\title{
Die Psychiatrische Rehabilitation wird zu einem der Zukunftsthemen der Psychiatrie - Pro \& Kontra
}

\author{
Psychiatric Rehabilitation will be a Future Topic for Psychiatry - Pro \& Contra
}

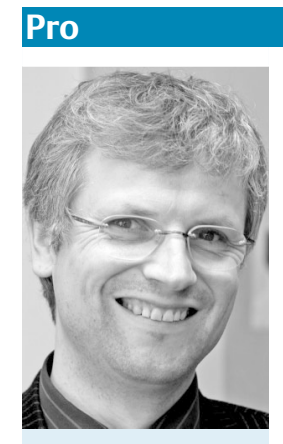

Matthias

Weisbrod

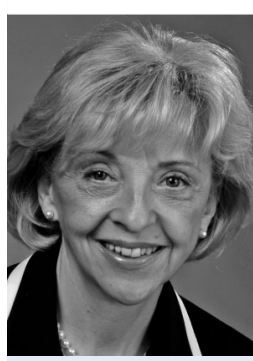

Regine

Müllensiefen
Ausgangslage

Psychiatrische Er-

krankungen verlau-

fen überwiegend chronisch und gefährden die soziale Teilhabe. Das Ausmaß sozialer Integration beeinflusst den Krankheitsverlauf wesentlich. Soziale Integration ist ein überragendes Behandlungsziel der betroffenen Menschen. Während der Mangel an qualifizierten Arbeitskräften eine unserer größten gesellschaftlichen Herausforderungen darstellt, nehmen Arbeitsunfähigkeitszeiten und Frühberentungen

aufgrund psychischer Erkrankungen zu und führen zu erheblichen Belastungen der sozialen Sicherungssysteme. Psychiatrische Rehabilitation leistet erfolgreich soziale und berufliche Integration. Sie erfüllt damit nicht nur ein zentrales Patientenanliegen, sondern trägt maßgeblich zur Lösung aktueller gesellschaftlicher Herausforderungen bei.

\section{Die Perspektive der Erkrankten}

Psychiatrische Erkrankungen beginnen überwiegend in der Adoleszenz und beeinträchtigen die soziale Entwicklung. Die resultierende soziale Störung verschlechtert den Krankheitsverlauf. Patienten in Arbeit werden schneller gesund [1], psychisch kranke Menschen in stabilen sozialen Verhältnissen erleiden weniger häufig akute Exazerbationen. Soziale und vor allem berufliche Integration stellen für psychisch kranke Menschen das wichtigste Behandlungsziel dar [2]. Psychiatrische Rehabilitation zielt direkt auf dieses zentrale Anliegen. Die Integrationsquoten psychiatrischer Rehabilitationseinrichtungen stellen eindrucksvoll unter Beweis, dass ein guter Teil der Betroffenen durch ressourcenorientierte, individuell passende Rehabilitationsmaßnahmen sozial und beruflich wiedereingegliedert werden kann.

\section{Die Behandlung}

Drastisch verkürzte Behandlungszeiten [3] haben die stationäre Behandlung zunehmend auf die Reduktion der Akutsymptomatik eingeengt. Das pauschalierende Entgeltsystem wird diese Entwicklung weiter zuspitzen. Die langfristige Entwicklung der Alltagsfunktionalität psychiatrisch kranker Menschen hängt aber nicht von der Akutsymptomatik ab. Vielmehr sind soziale Einbindung und kognitive Leistungsfähigkeit die den Verlauf bestimmenden Faktoren. Zur Erreichung dauerhafter Stabilität ist für psychisch kranke Menschen gesellschaftliche Teilhabe und eine Sinn und Halt gebende Struktur durch eine (leidensgerechte) (Arbeits-)Tätigkeit essenziell. Die Behandlung psychisch erkrankter Menschen muss daher - nicht zuletzt um für die Behandler befriedigend zu sein - im Kern rehabilitativ angelegt sein. Psychiatrische Rehabilitation kann nicht nur hinsichtlich Teilhabe bedeutsame Erfolge vorweisen, sie hat darüber hinaus einen positiven Einfluss auf Lebensqualität und Psychopathologie [4], so bessert sie z.B. bei an Schizophrenie erkrankten Menschen auch kognitive Leistungsfähigkeit und Negativsymptomatik [5].

\section{Die Perspektive der Gesellschaft}

Mangel an qualifizierten Arbeitskräften stellt eine unserer größten gesellschaftlichen Herausforderungen dar. Psychische Erkrankungen stehen bei Krankschreibung und Frühberentung inzwischen an erster Stelle, durch sie verursachte Arbeitsunfähigkeitstage sind gegen den Trend dramatisch angestiegen. Diese Entwicklung ist nicht nur auf Deutschland be- schränkt [6]. Hubert Seiter - der Vorsitzende der Geschäftsführung der DRV Baden-Württemberg - konstatiert 2010 [7], dass „brennend aktuelle Themen wie der demografische Wandel, der Fachkräftemangel, Arbeiten bis 67 gar nicht anders bewältigt werden können, als mit qualifizierter Rehabilitation“. Rehabilitation vermindert direkte Krankheitskosten und verbessert die Erwerbstätigenquote. Jeder in Rehabilitation investierte Euro rechnet sich mit dem Faktor 5 [8]. Angesichts der sozialpolitischen Bedeutung psychischer Erkrankungen ist insbesondere die Investition in eine weiter verbesserte psychiatrische Rehabilitation ökonomisch sinnvoll.

\section{Die Entwicklung psychiatrischer} Rehabilitation

Beeinträchtigungen des sozialen Funktionsniveaus finden sich bei Menschen mit unterschiedlichen psychiatrischen Erkrankungen $[9,10]$. Kognitive, interaktionelle und dynamische Defizite sowie mangelnde Fähigkeiten zur Selbststrukturierung haben sich als allgemein limitierende Faktoren für eine soziale und berufliche Teilhabe erwiesen. Diese Faktoren können durch konsequente Milieutherapie und Fremdstrukturierung in der Rehabilitation gebessert werden. Störungsspezifische Rehabilitationskonzepte, die diese Ansätze ergänzen, sind bislang nur in Ansätzen entwickelt.

Wir wissen um die überragende Anpassungsfähigkeit unseres Gehirns. Die Grundlagen der Neuroplastizität verstehen wir weit besser als die Neurochemie oder Genetik, die der Psychopathologie zugrunde liegen. Wir sollten das Verständnis des zentralen Prozesses, der Rehabilitation zugrunde liegt, zur Verbesserung spezifisch psychiatrischer Rehabilitationskonzepte nutzen [11].

Rehabilitation muss bei psychiatrischen Patienten als integrierter Bestandteil des Gesamtbehandlungsplans bereits in der Akutphase angelegt sein und kostenträgerbedingte Sektorengrenzen überspan- 
nen. Sie muss Teil der Frühbehandlung sein, da soziale Auswirkungen psychischer Erkrankungen i. allg. vor der klinischen Erstmanifestation einsetzen. Angesichts der steigenden Anforderungen der Gesellschaft an ältere Menschen muss sie bis ins höhere Alter greifen. Unsere Aufgabe ist es, die bewährten Rehabilitationskonzepte in diesem Sinne weiterzuentwickeln, Rehabilitationsdiagnostik auf breiter Ebene zu verfeinern und Menschen mit psychischen Erkrankungen individuell abgestimmte Reha-Maßnahmen zukommen zu lassen.

Fazit

Psychiatrische Rehabilitation muss eine bedeutende Rolle in Klinik, Behandlungsorganisation und Forschung einnehmen, da sie wesentliche Bedürfnisse psychiatrischer Patienten erfüllt, den Behandlern einen Weg aus den frustrierenden Mühlen einer Drehtürpsychiatrie weist, die Sozialsysteme nachhaltig entlastet und damit medizinisch, ethisch und ökonomisch sinnvoll ist.

Rehabilitation bezeichnet in einer anderen Bedeutung jene Aktionen, die geeignet sind, das Ansehen und den Ruf einer Person oder einer Personengruppe wiederherzustellen. Wir sollten psychiatri- sche Rehabilitation auch deshalb zu einem zentralen Schwerpunktthema machen, da sie geeignet ist, das Ansehen psychiatrisch erkrankter Menschen und damit auch das Ansehen unseres Faches zu heben.

\section{Literatur}

1 Warner R. Recovery from schizophrenia and the recovery model. Curr Opin Psychiatry 2009; 22: 374-380

2 Ramsay CE, Broussard B, Goulding SM et al. Compton life and treatment goals of individuals hospitalized for first-episode nonaffective psychosis. Psych Res 2011; 189 : 344-348

3 Baden-Württembergische Krankenhausgesellschaft (BWKG). Basisdaten des Gesundheitswesens. 2011: www.bwkg.de/down load/statistiken.html

4 Watzke S, Galvao A, Brieger P. Vocational rehabilitation for subjects with severe mental illnesses in Germany. A controlled study. Soc Psychiatry Psychiatr Epidemiol 2009; 44: 523-531

5 Bio DS, Gattaz WF. Vocational rehabilitation improves cognition and negative symptoms in schizophrenia. Schizophr Res 2011; 126 : 265-269

6 Henderson M, Harvey SB, Overland $S$ et al. Work and common psychiatric disorders. J R Soc Med 2011; 104: 198-207

7 Seiter $H$. Rehabilitation: Reha ist keine Last. Dtsch Ärztebl 2010; 107: A-2337
8 Steiner $M$, Zwingmann C, Riedel $W$ et al. Die medizinische Rehabilitation Erwerbstätiger - Sicherung von Produktivität und Wachstum. Basel, Berlin, Bremen, Brüssel, Düsseldorf: Prognos AG; 2009

9 Mao AR, Brams M, Babcock $T$ et al. A physician's guide to helping patients with ADHD find success in the workplace. Postgrad Med 2011; 123: 60 - 70

10 Zanarini MC, Frankenburg FR, Reich DB et al. The 10-year course of psychosocial functioning among patients with borderline personality disorder and axis II comparison subjects. Acta Psychiatr Scand 2010; 122: $103-109$

11 Vinogradov S, Fisher $M$, de Villers-Sidani $E$. Cognitive training for impaired neural systems in neuropsychiatric illness. Neuropsychopharmacology 2011; 37: 523-531

\section{Korrespondenzadresse}

\section{Prof. Dr. Matthias Weisbrod}

Abteilung für Psychiatrie und Psychotherapie, SRH Klinikum Karlsbad-Langensteinbach Guttmannstraße 1

76307 Karlsbad-Langensteinbach matthias.weisbrod@kkl.srh.de

\section{Bibliografie}

Dol http://dx.doi.org/

10.1055/s-0032-1305112

Psychiat Prax 2012; 39: 316-317

(c) Georg Thieme Verlag KG

Stuttgart · New York

ISSN 0303-4259 